Arq. Bras. Med. Vet. Zootec., v.68, n.1, p.215-223, 2016

\title{
Influência do horário de aplicação e da variedade genética em fêmeas de tilápias Oreochromis niloticus submetidas à indução hormonal com hCG
}

\author{
[Influence of application time and genetic variability in tilapias Oreochromis niloticus \\ submitted to hormonal induction with hCG] \\ U.N. Souza ${ }^{1}$, V.O. Felizardo ${ }^{1 *}$, R.T.F. Freitas ${ }^{1}$, C.C.V. Melo', \\ M.R. Ferreira ${ }^{2}$, R.V. Reis-Neto ${ }^{1}$ \\ ${ }^{1}$ Universidade Federal de Lavras - UFLA - Lavras, MG \\ ${ }^{2}$ Pontifícia Universidade Católica - Goiânia, Goiás
}

\begin{abstract}
RESUMO
Objetivou-se com este trabalho avaliar a influência do horário de aplicação e da variedade genética de fêmeas submetidas à indução com hCG. O experimento foi realizado nos meses de julho e agosto de 2012. Utilizou-se delineamento inteiramente ao acaso, com esquema fatorial $2 \times 4 \times 2$ (duas variedades, quatro horários de aplicação, com e sem hCG), sendo quatro repetições para os tratamentos controle e seis para induzidos com hCG. Foram utilizadas 40 fêmeas das variedades GIFT e UFLA, microchipadas, alojadas em um sistema com recirculação de água. Foram utilizados quatro horários de aplicação: seis; 12; 18 e 24 horas. A dosagem de hCG foi de 5UI/grama de peso de peixe, dividida em duas aplicações. A extrusão dos ovócitos foi realizada 720 horas-graus após a última aplicação, sendo observado o número de animais de cada tratamento que apresentaram desova. Os dados obtidos foram submetidos à análise de variância, e as médias comparadas pelo teste SNK a $5 \%$ de significância. A indução com hCG proporcionou melhores $(\mathrm{P}<0,05)$ resultados paras as variáveis: índice de desova (ID), fecundidade absoluta relativa ao comprimento (FARC) e peso de desova (PD), independentemente da variedade utilizada. A variedade UFLA não foi influenciada pelo horário de aplicação $(\mathrm{P}>0,05)$. Já a GIFT apresentou maior ID $(\mathrm{P}<0,05)$ quando a indução hormonal foi realizada às $24 \mathrm{~h}$ em relação à aplicação realizada às $18 \mathrm{~h}$. $\mathrm{O}$ diâmetro do ovócito da variedade UFLA é maior do que o da GIFT $(\mathrm{P}<0,05)$. Ao se observar o grupo controle, verificou-se que a variedade UFLA apresentou maior porcentagem de ovócitos com posição periférica de vesícula germinativa $(\mathrm{P}<0,05)$ em relação à GIFT. A indução hormonal com hCG foi influenciada pela variedade e pelo horário de aplicação.
\end{abstract}

Palavras-chave: frequência absoluta, qualidade ovocitária, reprodução induzida

\begin{abstract}
The aim of this work was to evaluate the influence of the application time and genetic variability of females submitted to hormonal induction with hCG. The experiment was performed in the months of July and August 2012. We used a completely randomized design, with a $2 \times 4 \times 2$ (two varieties, four application times, with or without hCG) factorial scheme, with four replicates for the control treatments and six replicated for those with hCG induction. Forty females of the GIFT and UFLA varieties, with microchips, were housed in a water recirculation system. Four application times were used: 6; 12; 18 and 24 hours. The hCG dosage was $5 U I / g r a m$ of fish, divided into two applications. Oocyte extrusion was performed 720 hours after the last application, observing the number of animals in each treatment which presented spawning. The obtained data were submitted to variance analysis and the means compared by the SNK test at 5\% significance. The induction with $h C G$ provided better $(P<0.05)$ results for the variables: spawning index $(S I)$, absolute fecundity relative to length (AFRL) and spawning weight (SW), regardless of the variety used. The UFLA variety was not influenced by the time of application $(P>0.05)$, while GIFT presented higher SI $(P<0.05)$ when the hormonal induction was performed at $24 \mathrm{~h}$ in relation to the application performed at $18 \mathrm{~h}$. The diameter of the oocytes of the UFLA variety was larger than that of the GIFT variety $(P<0.05)$. Observing the control group, the UFLA
\end{abstract}

Recebido em 29 de maio de 2014

Aceito em 27 de agosto de 2015

*Autor para correspondência (corresponding author)

E-mail: viviofbio@yahoo.com.br 
variety presented a higher percentage of oocytes with peripheral position of the germinal vesicle $(P<0.05)$ in relation to GIFT. The hormonal induction with hCG was influenced by the variety and the time of application.

Keywords: absolute frequency, oocyte quality, inducted reproductions

\section{INTRODUÇÃO}

A tilápia possui características reprodutivas favoráveis aos programas de melhoramento genético, como alta prolificidade, maturidade sexual precoce, fecundidade relativa elevada e desova parcelada. Entretanto, isso faz com que o controle reprodutivo seja um dos maiores desafios na tilapicultura (Turra et al., 2010).

Os programas de melhoramento genético são importantes para o desenvolvimento da tilapicultura não só para atender a crescente demanda mundial por pescado, mas também para reduzir os custos de produção, melhorar a resistência a doenças, o aproveitamento alimentar e a qualidade dos produtos (Gjedrem, 2000).

Para o sucesso de uma piscicultura de produção de alevinos, a qualidade dos ovos e o número de desovas é um ponto importante, pois resultarão em altas taxas de fertilização, eclosão e maior sobrevivência das larvas após a absorção do saco vitelino (Bromage e Cumaranatunga, 1992).

O uso da técnica de aplicação hormonal em peixes possibilita programar a reprodução de forma a obter resultados favoráveis no processo de seleção de animais e origem deles. As induções químicas podem ainda ser utilizadas para antecipar o período reprodutivo, restringi-lo ou mesmo sincronizar a reprodução de um lote de matrizes, o que possibilita ao produtor obter alevinos em épocas em que a lucratividade seja maior, ou em que o cultivo seja finalizado em período no qual a comercialização seja otimizada (Venturieri e Bernardino, 1999).

Uma série de mecanismos de ajuste está envolvida no processo de maturação gonadal e de desova, basicamente por meio de controles hormonais. No início do desenvolvimento gonadal, ocorre um aumento de concentração de gonadotrofinas na hipófise e no plasma, servindo provavelmente para recrutar os ovócitos e iniciar a vitelogênese no período reprodutivo corrente (Murgas et al., 2009).
Em se tratando da técnica de reprodução induzida artificialmente, esta já tem sido utilizada em tilápias para garantir o horário exato da fertilização e já foi realizada por Senthilkumaran et al. (2002), que induziram a desova de $O$. niloticus usando diferentes agentes indutores, como EBHC, LH-RH, hCG e um antagonista da dopamina. (Valentin, 2007).

Diante disso, o objetivo com o presente estudo foi avaliar a influência do horário de aplicação e da variedade genética em fêmeas de tilápias-donilo submetidas à indução hormonal com hCG.

\section{MATERIAL E MÉTODOS}

O experimento foi realizado no Laboratório de Recirculação da Estação de Piscicultura do Departamento de Zootecnia (DZO) da Universidade Federal de Lavras, UFLA - MG, Brasil, durante os meses de julho e agosto de 2012. Foram utilizadas 80 fêmeas de tilápias nilóticas $O$. niloticus, oriundas da Estação de Piscicultura do DZO - UFLA; destas, 40 eram reprodutoras da variedade GIFT, com idade média em torno de dois anos, com peso médio de $0,42 \pm 0,07 \mathrm{~kg}$ e comprimento médio de $21,00 \pm 1,30 \mathrm{~cm}$, e 40 eram da variedade UFLA, com idade média de quatro anos, pesando $0,61 \pm 0,12 \mathrm{~kg}$ e comprimento total médio de $24,63 \pm 1,78 \mathrm{~cm}$.

As matrizes se encontravam em tanques escavados, agrupadas por sexo, em ambiente natural, com temperatura média de $17,9^{\circ} \mathrm{C}$. As fêmeas estavam em repouso reprodutivo, sendo alimentadas duas vezes ao dia, com ração extrusada contendo $32 \%$ de proteína bruta.

As fêmeas aptas a receberem a indução hormonal foram selecionadas mediante observação das características secundárias de maturação sexual como: abdômen abaulado e macio ao toque, papila urogenital proeminente e de coloração rosada ou avermelhada e orifício genital ligeiramente aberto.

Após a identificação de cada fêmea por meio da leitura do microchip, localizado próximo à 
nadadeira dorsal esquerda, elas foram pesadas, seu comprimento total foi medido, sendo esses dados registrados em fichas e os animais acondicionados no Laboratório.

As fêmeas foram distribuídas em um delineamento inteiramente ao acaso, com oito caixas de polietileno com capacidade individual de 500 litros d'água, mantidas na proporção de 10 animais/caixa, separadas de acordo com as variedades. Cada caixa de polietileno apresentava fluxo de água e aeração contínua. A temperatura da água era mantida em $27,7 \pm 0,1^{\circ} \mathrm{C}$, os níveis de oxigênio dissolvido em $6,6 \pm 1,0 \mathrm{mg} . \mathrm{L}^{-1}$ e o $\mathrm{pH}$ em $6,7 \pm 0,1$.

No período experimental, que compreendeu 42 dias, os animais foram submetidos a um fotoperíodo de $12 \mathrm{~h} 12 \mathrm{~min}$, horas de luz:escuro (L:E) e alimentados com ração comercial extrusada contendo $36 \%$ de proteína bruta e pellets de dois milímetros de diâmetro, fornecida uma vez ao dia ad libitum. Antes do início da indução, as fêmeas permaneceram em jejum por um período de 24 horas, sendo observadas periodicamente. Nenhuma apresentou desova espontânea, porém, ao se aproximar o fim do período experimental, pôde-se notar uma evolução nas características reprodutivas.

As matrizes, após o jejum, foram induzidas artificialmente por via intramuscular com gonadotrofina coriônica humana (hCG) (Chorulon 5000UI, Laboratório Intervet SheringPlough Animal Health), com a aplicação realizada na base da nadadeira dorsal com dose total de 5UI por grama de peso, dividida em duas aplicações, a primeira correspondendo a $10 \%$ da dose total e a segunda de $90 \%$, aplicada 18 horas após a primeira dose, de acordo com a metodologia utilizada por Valentin (2007).

Durante os horários determinados, foram induzidas seis fêmeas de cada variedade com o hCG e em quatro fêmeas foram aplicadas duas doses de soro fisiológico, sendo estas, destinadas ao tratamento controle. Os momentos de injeção do hCG e soro fisiológico foram distribuídos em quatro sistemas de aplicação, sendo: sistema 1: 6/24h ( $1^{\mathrm{a}}$ dose $/ 2^{\mathrm{a}}$ dose $)$; sistema 2: 12/6h; sistema 3: $18 / 12 \mathrm{~h}$ e sistema $4: 24 / 18$ horas.

Após 24 horas da aplicação da segunda dose, os animais foram retirados de suas caixas individualmente, a papila urogenital e as superfícies circunjacentes limpas e enxugadas com toalha de papel e, em seguida, submetidas à massagem abdominal com suave movimento anteroposterior, para a extrusão de gametas.

Logo após a coleta e pesagem da desova, foi destinado $1 \mathrm{~g}$ de ovócitos para determinação do número de ovócitos/grama. O diâmetro $(\mu \mathrm{m})$ aferido em 10 ovócitos de cada fêmea, previamente imersos em solução de Gilson $(5 \mathrm{~mL}$ de álcool 60\%, $44 \mathrm{~mL}$ de água destilada, 0,7g de ácido nítrico $80 \%, 1 \mathrm{~g}$ de cloreto de mercúrio e $0,9 \mathrm{~mL}$ de ácido acético glacial).

Para a análise da posição periférica da vesícula germinativa (PPVG \%), 25 ovócitos de cada fêmea foram imersos em solução de Serra $(60 \mathrm{~mL}$ de álcool 90\%, 30mL de formaldeído e $10 \mathrm{~mL}$ de ácido acético glacial). As análises de diâmetro e PPVG foram realizadas com auxílio de microscópio óptico de 40x Vazzoler (1996).

Após verificar a quantidade de ovócitos/g de peixe, foram realizados os seguintes cálculos: índice de desova (ID= $(\mathrm{PD} / \mathrm{PF}) \times 100)$, em que: $\mathrm{PD}$ $=$ peso da desova e $\mathrm{PF}=$ peso da fêmea subtraindo-se o PD; fecundidade absoluta ( $\mathrm{FA}=\mathrm{PD} \times$ número de ovócitos em $1 \mathrm{~g}$ ), o que resulta no número total de ovócitos produzidos por peixe; fecundidade absoluta relativa para peso $(\mathrm{FARP}=(\mathrm{FA} / \mathrm{PT}))$, sendo o número total de ovócitos por grama do exemplar, em que PT $=$ peso total do animal, e fecundidade absoluta relativa para comprimento $(\mathrm{FARC}=\mathrm{FA} / \mathrm{CT})$ equivale ao número de ovócitos por centímetro do animal, em que $\mathrm{CT}=$ comprimento total do animal.

Os parâmetros reprodutivos foram avaliados pelas metodologias usadas por Coward e Bromage (1999a, 1999b) e Godinho (2007).

Os dados obtidos das variáveis reprodutivas foram previamente testados quanto às pressuposições e submetidos à análise de variância por meio de um modelo estatístico que considerou as variedades de tilápias testadas (UFLA e GIFT), a aplicação ou não de hormônio e a interação entre essas variáveis, além dos horários de aplicação hormonal aninhados às variedades. Quando foi detectada diferença entre os horários, as médias foram comparadas pelo teste SNK adotando-se o nível de $5 \%$ de 
significância. Todas as análises foram realizadas utilizando-se o software " $\mathrm{R}$ " para Windows na versão 2.13.2.

Este projeto foi aprovado pelo Comitê de Ética da Universidade Federal de Lavras, com número de protocolo: 016-13.

\section{RESULTADOS E DISCUSSÃO}

A variedade UFLA respondeu melhor quando induzida com o hormônio hCG, visto que 87,5\% das fêmeas induzidas desovaram, enquanto o grupo controle apresentou somente 56,3\% de fêmeas desovadas. Por outro lado, a variedade GIFT parece não ser influenciada pela aplicação do hormônio hCG, sendo observada porcentagem semelhante de fêmeas desovadas entre o grupo controle e o grupo induzido (Tab. 1). Esses resultados indicam um ponto positivo da variedade UFLA em relação à GIFT, no que diz respeito à reprodução para programas de melhoramento genético.

O número de peixes que desovaram após a indução com hCG foi diferente quando comparados os horários de aplicação do hormônio. $\mathrm{Na}$ Tab. 1, é possível observar que $100 \%$ das tilápias da variedade UFLA, tanto no horário de aplicação seis horas quanto no de $12 \mathrm{~h}$, desovaram. Porém, nos horários noturnos de $18 \mathrm{e}$ 24 horas de aplicação do hCG, apenas $67 \%$ e $83 \%$ das fêmeas desovaram, respectivamente.

Quando foi avaliada a porcentagem total do número de fêmeas desovadas independentemente da aplicação hormonal ou não, foi observada maior porcentagem de fêmeas que desovaram no horário em que a primeira aplicação foi realizada às 18 horas $(85 \%)$, enquanto os animais que receberam a primeira aplicação às 12 horas apresentaram a menor porcentagem de fêmeas desovadas (55\%) (Tab. 1).

Tabela 1. Tilápias das variedades GIFT e UFLA que desovaram com e sem indução hormonal

\begin{tabular}{ccccccc}
\hline $\begin{array}{c}\text { Horário de } \\
\text { aplicação }\end{array}$ & $\begin{array}{c}\text { UFLA sh } \\
(\mathrm{n}=4)\end{array}$ & $\begin{array}{c}\text { UFLA ch } \\
(\mathrm{n}=6)\end{array}$ & $\begin{array}{c}\text { GIFT sh } \\
(\mathrm{n}=4)\end{array}$ & $\begin{array}{c}\text { GIFT ch } \\
(\mathrm{n}=6)\end{array}$ & $\begin{array}{c}\text { Total } \\
(\mathrm{n}=20)\end{array}$ & Total (\%) \\
\hline 6 & 1 & 6 & 3 & 5 & 15 & 75 \\
12 & 1 & 6 & 1 & 3 & 11 & 55 \\
18 & 3 & 4 & 4 & 6 & 17 & 85 \\
24 & 4 & 5 & 3 & 3 & 15 & 75 \\
\hline Total (n) & 9 & 21 & 11 & 17 & & \\
Total (\%) & 56,3 & 87,5 & 68,8 & 70,8 & & \\
\hline
\end{tabular}

(sh: sem hormônio, ch: com hormônio) em diferentes horários de indução.

Ao se avaliarem os resultados acima, pode-se observar que existe uma tendência de as tilápias da variedade UFLA desovarem em maior quantidade no período em que a primeira aplicação ocorreu no início e na metade do dia. A indução hormonal em tilápias vem sendo utilizada para garantir o horário exato da fertilização, facilitando a manipulação e fertilização de gametas em programas de seleção genética. Diferentes indutores já tem sido utilizados, como LH-RH, antagonista da dopamina e hCG (Mylonas et al., 2010; Senthilkumaran et al., 2002).

Foi possível observar, na variedade GIFT no grupo controle, um alto número de ovócitos por grama de desova (Fig. 1). Esse resultado é um fator negativo na reprodução, visto que um alto número de ovócitos está relacionado com ovócitos de menor diâmetro, que provavelmente não são considerados maduros.

O índice de desova (ID) representa o número de ovócitos liberados em relação ao peso do animal. Quando foram avaliados os resultados, foi possível observar que, para a variedade UFLA, independentemente da utilização ou não do hCG, o horário de aplicação não influenciou $(\mathrm{P}>0,05)$ essa variável. Já para a variedade GIFT, o horário de aplicação do hormônio influenciou $(\mathrm{P}<0,05)$ esse parâmetro (Tab. 2). 
Influência do horário...

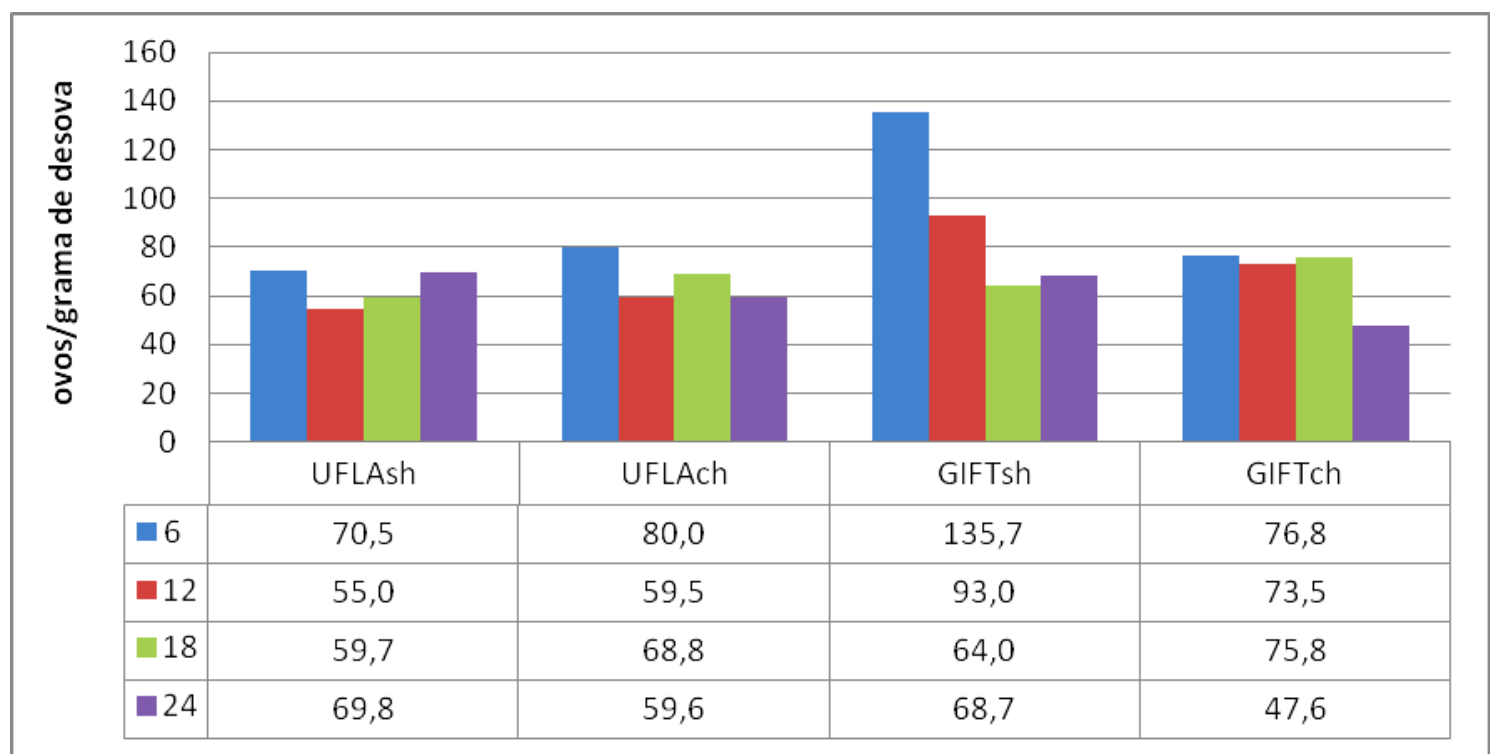

Figura 1. Relação do número de ovócitos por grama de desova nas variedades UFLA e GIFT de tilápia sem (sh) e com (ch) aplicação do hormônio hCG.

Tabela 2. Médias e desvios-padrão dos parâmetros reprodutivos nas tilápias variedade UFLA nos diferentes horários de indução

\begin{tabular}{|c|c|c|c|c|c|c|c|c|c|c|c|c|}
\hline \multirow{3}{*}{$\begin{array}{l}\text { Hor. } \\
\text { Aplic. }\end{array}$} & \multicolumn{12}{|c|}{ Variáveis } \\
\hline & \multicolumn{2}{|c|}{ ID } & \multicolumn{2}{|c|}{ FA } & \multicolumn{2}{|c|}{ FARP } & \multicolumn{2}{|c|}{ FARC } & \multicolumn{2}{|c|}{ DIAM } & \multicolumn{2}{|c|}{ PPVG } \\
\hline & $\mathrm{ch}$ & $\mathrm{Sh}$ & $\mathrm{ch}$ & sh & $\mathrm{ch}$ & $\mathrm{Sh}$ & $\mathrm{ch}$ & sh & ch & sh & ch & sh \\
\hline 6 & $\begin{array}{c}1,2 \pm \\
1,1\end{array}$ & - & $\begin{array}{c}526,9 \pm \\
477,3\end{array}$ & - & $\begin{array}{c}1,0 \pm \\
0,9\end{array}$ & - & $\begin{array}{c}22,7 \pm \\
20,5\end{array}$ & - & $\begin{array}{c}3854,6 \pm \\
686,4\end{array}$ & - & $\begin{array}{c}0,4 \pm \\
0,3\end{array}$ & - \\
\hline 12 & $\begin{array}{c}1,6 \pm \\
0,3\end{array}$ & - & $\begin{array}{c}680,1 \pm \\
233,3\end{array}$ & - & $\begin{array}{c}1,0 \pm \\
0,3\end{array}$ & - & $\begin{array}{c}26,5 \pm \\
9,1\end{array}$ & - & $\begin{array}{c}4199,3 \pm \\
409,0\end{array}$ & - & $\begin{array}{c}0,6 \pm \\
0,2\end{array}$ & - \\
\hline 18 & $\begin{array}{c}2,1 \pm \\
0,2\end{array}$ & $\begin{array}{c}0,8 \pm \\
1,1\end{array}$ & $\begin{array}{c}814,8 \pm \\
299,9\end{array}$ & $\begin{array}{c}487,7 \pm \\
652,2\end{array}$ & $\begin{array}{c}1,4 \pm \\
0,4\end{array}$ & $\begin{array}{c}0,5 \pm \\
0,7\end{array}$ & $\begin{array}{c}34,6 \pm \\
12,1\end{array}$ & $\begin{array}{c}17,3 \pm \\
23,3\end{array}$ & $\begin{array}{c}3934,0 \pm \\
566,8\end{array}$ & $\begin{array}{l}4381,3 \\
\pm 97,5 \mathrm{a}\end{array}$ & $\begin{array}{c}0,8 \pm \\
0,2\end{array}$ & $\begin{array}{c}0,9 \pm \\
0,1\end{array}$ \\
\hline 24 & $\begin{array}{c}1,8 \pm \\
0,4\end{array}$ & $\begin{array}{c}1,1 \pm \\
0,1\end{array}$ & $\begin{array}{c}736,7 \pm \\
297,5\end{array}$ & $\begin{array}{c}727,6 \pm \\
153,7\end{array}$ & $\begin{array}{c}1,1 \pm \\
0,3\end{array}$ & $\begin{array}{c}0,7 \pm \\
0,1\end{array}$ & $\begin{array}{c}28,3 \pm \\
11,0\end{array}$ & $\begin{array}{c}24,1 \pm \\
5,2\end{array}$ & $\begin{array}{c}4084,8 \pm \\
302,8\end{array}$ & $\begin{array}{c}3734,0 \\
\pm 205,7 \\
\text { b }\end{array}$ & $\begin{array}{c}0,4 \pm \\
0,4\end{array}$ & $\begin{array}{c}0,7 \pm \\
0,5\end{array}$ \\
\hline
\end{tabular}

Letras minúsculas diferem na coluna, indicando diferença estatística a $5 \%$ de probabilidade pelo teste de SNK. Horário de aplicação (Hor. Aplic.), índice de desova (ID), fecundidade absoluta (FA), fecundidade absoluta relativa ao peso (FARP) e fecundidade absoluta relativa ao comprimento (FARC), diâmetro do ovócito (DIAM) e posição periférica da vesícula germinativa (PPVG), nas variedades UFLA e GIFT induzidas (ch) ou não (sh) com hCG.

Os animais da variedade GIFT que receberam a indução às 24 horas apresentaram maior $(\mathrm{P}<0,05)$ ID em relação aos animais que foram induzidos às 18 horas, não diferindo dos demais horários. No entanto, no grupo controle, os animais induzidos às 18 horas foram os que apresentaram maior ID $(\mathrm{P}<0,05)$ em relação aos induzidos às seis e às 24 horas (Tab. 3). Esse resultado pode ser devido ao preparo prévio dos animais para reprodução. Isso porque, embora tenha sido realizada uma seleção mediante visualização de características externas dos animais, essa seleção pode ser subjetiva, levando a selecionar animais que não se encontram totalmente preparados reprodutivamente (Tab. 2 e 3).

Já as fecundidades absoluta, absoluta relativa ao peso e ao comprimento, bem como a posição periférica de vesícula germinativa, não apresentaram diferença estatística $(\mathrm{P}>0,05)$ em relação aos diferentes horários de aplicação nas variedades UFLA e GIFT (Tab. 2 e 3).

$\mathrm{Na}$ variedade UFLA, o diâmetro dos ovócitos do grupo controle apresentou-se maior $(\mathrm{P}<0,05)$ quando recebeu a aplicação às 18 horas, em relação ao grupo aplicado às 24 horas (Tab. 2). 
Tabela 3. Médias e desvios-padrão dos parâmetros reprodutivos nas tilápias variedade GIFT nos diferentes horários de indução

\begin{tabular}{|c|c|c|c|c|c|c|c|c|c|c|c|c|}
\hline \multirow{3}{*}{$\begin{array}{l}\text { Hor } \\
\text { Aplic }\end{array}$} & \multicolumn{12}{|c|}{ Variáveis } \\
\hline & \multicolumn{2}{|c|}{ ID } & \multicolumn{2}{|c|}{$\mathrm{FA}$} & \multicolumn{2}{|c|}{ FARP } & \multicolumn{2}{|c|}{ FARC } & \multicolumn{2}{|c|}{ DIAM } & \multicolumn{2}{|c|}{ PPVG } \\
\hline & ch & sh & $\mathrm{Ch}$ & sh & ch & sh & $\mathrm{Ch}$ & $\mathrm{Sh}$ & ch & sh & ch & sh \\
\hline \multirow{2}{*}{6} & $1,8 \pm$ & $0,8 \pm$ & $401,8 \pm$ & $676,2 \pm$ & $1,2 \pm$ & $1,2 \pm$ & $20,1 \pm$ & $29,7 \pm$ & $3737,0 \pm$ & $3326,6 \pm$ & $0,7 \pm$ & $0,5 \pm$ \\
\hline & $1,0 \mathrm{ab}$ & $0,1 b$ & 207,5 & 386,4 & 0,7 & 0,7 & 10,7 & 16,3 & 212,9 & 514,0 & 0,3 & 0,3 \\
\hline \multirow{2}{*}{12} & $1,6 \pm$ & & $389.4 \pm$ & & $1,0 \pm$ & & $18,1 \pm$ & & $3932,0 \pm$ & & $0,5 \pm$ & \\
\hline & $1,1 \mathrm{ab}$ & - & 258.8 & - & 0.6 & - & 11.5 & $\begin{array}{ll}- \\
-\end{array}$ & 124.7 & - & 0,4 & $\begin{array}{ll}- \\
-\end{array}$ \\
\hline \multirow{2}{*}{18} & $0,6 \pm$ & $1,6 \pm$ & $220,3 \pm$ & $576,6 \pm$ & $0,5 \pm$ & $1,1 \pm$ & $10,3 \pm$ & $25,7 \pm$ & $3757,3 \pm$ & $3830,6 \pm$ & $0,4 \pm$ & $0,3 \pm$ \\
\hline & $0,2 b$ & $0,5 \mathrm{a}$ & 139,7 & 346,6 & 0,3 & 0,7 & 6,3 & 16,1 & 591,5 & 326,1 & 0,3 & 0,2 \\
\hline \multirow{2}{*}{24} & $2,2 \pm$ & $0,7 \pm$ & $477,2 \pm$ & $291,8 \pm$ & $1,3 \pm$ & $0,5 \pm$ & $23,7 \pm$ & $12,7 \pm$ & $3870,6 \pm$ & $3270,6 \pm$ & $0,1 \pm$ & $0,1 \pm$ \\
\hline & $0,1 \mathrm{a}$ & $0,5 b$ & 161,2 & 279,6 & 0,1 & 0,4 & 6,8 & 12,1 & 344,7 & 341,7 & 0,1 & 0,1 \\
\hline
\end{tabular}

Letras minúsculas diferem na coluna, indicando diferença estatística a $5 \%$ de probabilidade pelo teste de SNK. Horário de aplicação (Hor. Aplic.), índice de desova (ID), fecundidade absoluta (FA), fecundidade absoluta relativa ao peso (FARP) e fecundidade absoluta relativa ao comprimento (FARC), diâmetro do ovócito (DIAM) e posição periférica da vesícula germinativa (PPVG), nas variedades UFLA e GIFT induzidas (ch) ou não (sh) com hCG.

O índice de desova foi influenciado pela indução com hCG, sendo de $1,54 \%$ para as fêmeas induzidas e de $1,03 \%$ para as fêmeas não induzidas. Já a fecundidade absoluta não foi influenciada pela variedade de tilápia utilizada nem pela indução hormonal (Tab. 4).

Ao se avaliar a média do índice de desova das fêmeas, o grupo induzido com hCG apresentou maior $(\mathrm{P}<0,05)$ resultado que o grupo controle, independentemente da variedade utilizada (Tab. 4). Nascimento (2010) encontrou um índice de desova variando de $0,64 \%$ ate $1,04 \%$, sem utilização da indução hormonal, sendo esses resultados muito semelhantes ao encontrado para o grupo sem indução hormonal. Ao se avaliar o número de ovócito por grama (OVOG), pôde-se observar uma média de 67,2 para a variedade UFLA e de 77,4 para a variedade GIFT, sem que a indução hormonal afetasse essas variáveis.

A fecundidade absoluta também foi influenciada pelo hormônio, principalmente para a variedade GIFT. Nesta, a FA foi menor $(\mathrm{P}<0,05)$ para os peixes induzidos $(345,60)$ em relação ao grupo controle $(514,91)$. Porém, tal resultado somente foi encontrado para essa variedade (Tab. 4).

Quando avaliada a FARP (Tab. 4), foi observado que as fêmeas induzidas apresentaram maior $(\mathrm{P}<0,05)$ resultado, 1 quando comparadas com as fêmeas não induzidas, 0,79 , independentemente da variedade utilizada.

A FARC $(\mathrm{P}>0,05)$ não foi influenciada nem pela variedade nem pela indução hormonal (Tab. 4).
É importante salientar que a fecundidade pode variar de acordo com a variedade de tilápia utilizada (Neumann, 2004).

Ao se avaliar o PD (Tab. 4), pôde-se observar que este foi influenciado tanto pela indução hormonal quanto pela variedade de tilápia utilizada. As fêmeas GIFT apresentaram menores $(\mathrm{P}<0,05)$ valores de $\mathrm{PD}$ quando comparadas com as fêmeas UFLA. O aumento dessa variável nos peixes induzidos está relacionado com a capacidade do hCG em estimular a maturação folicular ovariana. De acordo com Zohar e Mylonas (2001), os hormônios indutores têm a capacidade de estimular a liberação de hormônio luteinizante, que, no final da vitelogênese, induz migração e desintegração da vesícula germinativa, rompimento do envelope folicular e liberação dos ovócitos na luz dos ovários (Zaniboni Filho e Weingartner, 2007), influenciando, assim, no aumento do peso da desova.

O diâmetro dos ovócitos foi maior $(\mathrm{P}<0,05)$ para a variedade UFLA $(4017,23 \mu \mathrm{m})$ quando comparado com o dos ovócitos das tilápias variedade GIFT $(3641,12 \mu \mathrm{m})$. O aumento do diâmetro pode influenciar na sobrevivência das larvas, indicando maior quantidade de reservas energéticas (Kjorsvik et al., 1990; BROOKS et al., 1997; Bonislawska et al., 2001). Já a aplicação do hCG não influenciou $(\mathrm{P}>0,05)$ o diâmetro, visto que as fêmeas induzidas apresentaram DIAM de 3914,64, enquanto sem hCG esse valor foi de $3743,41 \mu \mathrm{m}$. 
Influência do horário...

Tabela 4. Médias e desvios-padrão de parâmetros reprodutivos de diferentes variedades de tilápia nilótica

\begin{tabular}{|c|c|c|c|c|c|}
\hline Variáveis & Variedade & $\begin{array}{c}\text { Induzidas } \\
(\mathrm{n}=3 \text { a } 6)\end{array}$ & $\begin{array}{l}\text { Controle } \\
(\mathrm{n}=2 \text { a } 4)\end{array}$ & Média & $\mathrm{CV}$ \\
\hline \multirow{3}{*}{$\mathrm{PD}(\mathrm{g})$} & UFLA & $10,3 \pm 4,3$ & $9,22 \pm 5,7$ & $9,75 \mathrm{~A}$ & \multirow[t]{3}{*}{47,5} \\
\hline & GIFT & $5,35 \pm 3,12$ & $6,06 \pm 2,9$ & $5,70 \mathrm{~B}$ & \\
\hline & Média & $7,82 \mathrm{a}$ & $7,64 \mathrm{~b}$ & & \\
\hline \multirow{3}{*}{ ID $(\%)$} & UFLA & $1,67 \pm 0,6$ & $1,00 \pm 0,6$ & 1,33 & \multirow[t]{3}{*}{47,08} \\
\hline & GIFT & $1,41 \pm 0,9$ & $1,07 \pm 0,5$ & 1,24 & \\
\hline & Média & $1,54 \mathrm{a}$ & $1,03 \mathrm{~b}$ & & \\
\hline \multirow{3}{*}{$\begin{array}{l}\text { OVOG (nº de } \\
\text { ovócito em } 1 \mathrm{~g})\end{array}$} & UFLA & $67,14 \pm 36,45$ & $65,43 \pm 13,0$ & 66,2 & \multirow[t]{3}{*}{47,08} \\
\hline & GIFT & $67,37 \pm 21,97$ & $89,44 \pm 52,1$ & 78,4 & \\
\hline & Média & 67,25 & 77,43 & & \\
\hline \multirow{3}{*}{$\begin{array}{c}\text { FA (nº ovócitos } \\
\text { liberados) }\end{array}$} & UFLA & $675,51 \pm 336,5 \mathrm{~A}$ & $624,80 \pm 412,3$ & 650,15 & \multirow[t]{3}{*}{58,8} \\
\hline & GIFT & $345,60 \pm 196,2 \mathrm{Bb}$ & $514,91 \pm 341,7 \mathrm{a}$ & 430,25 & \\
\hline & Média & 510,55 & 569,85 & & \\
\hline \multirow{3}{*}{$\begin{array}{c}\text { FARP }\left(\mathrm{n}^{\mathrm{o}} \mathrm{de}\right. \\
\text { ovócito por } \mathrm{g} \text { de } \\
\text { peixe) }\end{array}$} & UFLA & $1,11 \pm 0,58$ & $0,67 \pm 0,47$ & 0,89 & \multirow[t]{3}{*}{58,4} \\
\hline & GIFT & $0,90 \pm 0,55$ & $0,91 \pm 0,62$ & 0,9 & \\
\hline & Média & $1,00 \mathrm{a}$ & $0,79 \mathrm{~b}$ & & \\
\hline \multirow{3}{*}{$\begin{array}{c}\text { FARC } \\
\left(\mathrm{n}^{\mathrm{o}} \text { ovócito/ }\right. \\
\text { comprimento de } \\
\text { peixe })\end{array}$} & UFLA & $27,42 \pm 13,8$ & $21,25 \pm 14,4$ & 24,33 & \multirow[t]{3}{*}{58,2} \\
\hline & GIFT & $16,70 \pm 9,49$ & $22,70 \pm 15,2$ & 19,70 & \\
\hline & Média & 22,06 & 21,97 & & \\
\hline \multirow{3}{*}{ DIAM $(\mu \mathrm{m})$} & UFLA & $4023,05 \pm 496,5$ & $4011,43 \pm 379,5$ & $4017,23 \mathrm{~A}$ & \multirow[t]{3}{*}{11,4} \\
\hline & GIFT & $3806,25 \pm 387,1$ & $3476,00 \pm 439,5$ & $3641,12 B$ & \\
\hline & Média & 3914,64 & 3743,41 & & \\
\hline \multirow{3}{*}{ PPVG (\%) } & UFLA & $55 \pm 34 b$ & $83 \pm 37 \mathrm{aA}$ & 69 & \multirow[t]{3}{*}{63,1} \\
\hline & GIFT & $45 \pm 37^{a}$ & $30 \pm 29 \mathrm{bB}$ & 37 & \\
\hline & Média & 50 & 56 & & \\
\hline
\end{tabular}

Nota: Letras maiúsculas diferem na coluna e letras minúsculas diferem na linha, indicando diferença estatística a 5\% de probabilidade pelo teste de SNK. Peso de desova (PD), índice de desova (ID), número de ovócitos por grama (OVOG), fecundidade absoluta (FA), fecundidade absoluta relativa ao peso (FARP) e fecundidade absoluta relativa ao comprimento (FARC), diâmetro do ovócito (DIAM) e posição periférica da vesícula germinativa (PPVG), nas variedades UFLA e GIFT induzidas ou não com hCG.

O diâmetro dos ovócitos pode ser utilizado como parâmetro de qualidade de desova (Bonislawska et al., 2000); para tilápias, esse valor varia entre 2,00 e 7,90mm (De Graaf et al., 1999). Neumann (2004) relata ovócitos com diâmetro entre 2,41 e 2,70mm, e Bern e Avtalion (1990) relata ovos de peixes adultos de tilápia-do-nilo com tamanho de 2,00 e $3,00 \mathrm{~mm}$, valores inferiores ao encontrado neste experimento, quando aplicado o hCG.

O hCG proporciona aumento dos níveis de gonadotrofinas, que estimulam o crescimento folicular e, consequentemente, os níveis de estradiol, que estão relacionados com a incorporação e a vitelogenina nos ovócitos (Davis et al., 2007). Esses ovos são maiores, proporcionando larvas maiores e com saco vitelino maior (Wootton, 1994; Gisbert et al., 2000) e maior sobrevivência larval. Assim, é possível concluir que a variedade UFLA proporcionou melhor diâmetro ovocitário, que está relacionado com a sobrevivência de larvas, o que seria uma qualidade requisitada na produção comercial. 
A variedade UFLA do grupo controle apresentou maior $(\mathrm{P}<0,05)$ porcentagem de $\mathrm{PPVG}$ em relação ao grupo que recebeu a indução hormonal. Provavelmente este resultado foi devido ao número de fêmeas que apresentaram liberação da desova, visto que do grupo induzido 21 fêmeas desovaram, e do grupo controle apenas 7, levando a uma menor variabilidade dessa avaliação. Por outro lado, na variedade GIFT, o grupo induzido apresentou maior $(\mathrm{P}<0,05)$ porcentagem de $\mathrm{PPVG}$ em relação ao grupo controle.

No grupo controle, a variedade UFLA apresentou maior $(\mathrm{P}<0,05)$ porcentagem de PPVG em relação à variedade GIFT. A posição periférica da vesícula germinativa é um indicador do grau de desenvolvimento gonadal, tendo em vista que os ovócitos com PPVG são considerados maduros (Narahara et al., 2002); este é um dos principais parâmetros que indica a qualidade do ovócito. Dessa forma, pode-se inferir que a variedade UFLA é mais indicada para a reprodução.

\section{CONCLUSÃO}

A indução hormonal com hCG foi influenciada pela variedade e pelo horário de aplicação. A variedade UFLA respondeu melhor à indução hormonal quando realizada nos horários de seis e de 12 horas.

\section{REFERÊNCIAS}

BERN, O.; AVTALION, R.R. Some morphological aspects of fertilization in tilapias. J. Fish Biol., v.36, p.375-381, 1990.

BONISLAWSKA, M.; FORMICKI, K.; KORZELECKA-ORKISZ, A.; WINNICKI, A. Fish egg size variability: biological significance. Fisheries, v.4, p.1-15, 2001.

BONISLAWSKA, M.; FORMICKI, K.; WINNICKI, A. Size of eggs and duration of embryogenesis in fishes. Acta Icht. Piscat., v.30, p.61-71, 2000.

BROMAGE, N.R.; CUMARANATUNGA, R. Egg production in the rainbow trout. In: MUIR, J. F.; ROBERTS, R. (Eds.). Recent advances in aquaculture. London: Croom Helm, 1992. p. 63138.
BROOKS, S.; TYLER, C.R.; SUMPTER, J.P. Egg quality in fish: what makes a good egg? Rev. Fish Biol. Fisher., v.7, p.387-416, 1997.

COWARD, K.; BROMAGE, N.R. Spawning frequency, fecundity, eggs size and ovarian histology in groups of Tilapia zillii maintained upon two distinct food ration sizes from firstfeeding to sexual maturity. Aquat. Living Resour., v.12, p.11-22, 1999a.

COWARD, K.; BROMAGE, N.R. Spawning periodicity, fecundity and egg size in laboratoryheld stocks of a substrate-spawning tilapiine, Tilapia zillii (Gervais). Aquaculture, v.171, p.251-267, 1999b.

DAVIS, L.K.; HIRAMATSU, N.; HIRAMATSU, $\mathrm{K}$ et al. Induction of three vitellogenins by 17 beta-estradiol with concurrent inhibition of the growth hormone-insulin-like growth factor 1 axis in a euryhaline teleost, the tilapia (Oreochromis mossambicus). Biol. Reprod., v.77, p.614-625, 2007.

DE GRAAF, G.J.; GALEMONI, F.; HUISMAN, E.A. Reproductive biology of pond-reared Nile tilapia, Oreochromis niloticus L. Aquac. Res., v.30, p.25-33, 1999.

GISBERT, E.; WILlIOT, P.; CASTELlÓORVAY, F. Influence of egg size on growth and survival ao early stages of Siberian sturgeon (Acipenser baeri) under small scale hatchery conditions. Aquaculture, v.183, p.83-94, 2000.

GJEDREM, T. Genetic improvement of coldwater species. Aquac. Res., v.31, p.25-33, 2000.

GODINHO, H.P. Reproductive strategies of fishes applied to aquaculture: bases for development of production technologies. Rev. Bras. Reprod. Anim., v.31, p.351-360, 2007.

KJORSVIK, E.; MANGOR-JENSEN, A.; HOLMEFJORD, I. Egg quality in fishes. $A d v$. Mar. Biol., v.26, p.71-113, 1990.

MURGAS, L.D.S.; DRUMOND, M.M.; PEREIRA, G.J.M. et al. Manipulação do ciclo e da eficiência reprodutiva em espécies nativas de peixes de água doce. In: CONGRESSO BRASILEIRO DE REPRODUÇÃO ANIMAL, 6., 2009, Belo Horizonte. Anais... Belo Horizonte: CBRA, 2009. p.74-80. 
MYLONAS, C.C.; FOSTIER, A.; ZANUY, S. Broodstock management and hormonal manipulations of fish reproduction. Gen. Comp. Endocrinol., v.165, p.516-534, 2010.

NARAHARA, M.Y.; TALMELLI, E.F.A.; KAVAMOTO, E.T.; GODINHO, H.M. Reprodução induzida da Pirapitinga-do-Sul, Brycon opalinus (Cuvier, 1819), mantida em condições de confinamento. Rev. Bras. Zootec., v.31, p.1070-1075, 2002.

NASCIMENTO, T. S. R. Vitamina E em dietas para reprodutoras de tilápia-do-nilo. 2010. 82f. Dissertação (mestrado em Aquicultura) Universidade Estadual Paulista, Jaboticabal, SP.

NEUMANN, E. Características do desenvolvimento inicial de duas linhagens de tilápia, Oreochromis niloticus e uma linhagem híbrida de Oreochromis sp. 2004. 63f. Dissertação (Mestrado em Aquicultura) Universidade Estadual Paulista, Jaboticabal, SP.

SENTHILKUMARAN, B.; SUDHAKUMARI, C.C.; CHANG, X.T. Ovarian carbonyl reductaselike 20b-hidroxysteroid dehydrogenase shows distinct surge in messenger RNA expression during natural and gonadotropin-induced meiotic maturation in Nile tilapia. Biol. Reprod., v.67, p.1080-1086, 2002.
TURRA E.M.; OLIVEIRA, D.A.A.; TEIXEIRA, E.A.. Uso de medidas morfométricas no melhoramento genético do rendimento de filé da tilápia do Nilo (Oreochromis niloticus). Rer. Bras. Reprod. Anim., v.34, p.29-36, 2010.

VALENTIN, F.N. Efeito da idade das matrizes de tilápia do nilo Oreochromis niloticus no desenvolvimento embrionário e larval. 2007. 43f. Dissertação (Mestrado em Aquicultura) Universidade Estadual Paulista, Jaboticabal, SP.

VAZZOLER, A.E.A.M. Biologia da reprodução de peixes teleósteos: teoria e prática. Maringá: EDUEM; São Paulo: SBI, 1996. 169 p.

VENTURIERI, R.; BERNARDINO, G. Hormônios na reprodução artificial de peixes. Pan. Aquicult., v.9, p.39-48, 1999.

WOOTTON, R.J. Life histories as sampling devices: optimum egg size in pelagic fishes. $J$. Fish Biol., v.45, p.1067-1077, 1994.

ZANIBONI FILHO, E; WEINGARTNER, M. Técnicas de indução da reprodução de peixes migradores. Rev. Bras. Rep. Anim., v.31, p.367373, 2007.

ZOHAR, Y.; MYLONAS, C.C. Endocrine manipulations of spawning in cultured fish: from hormones to genes. Aquaculture, v.197, p.99136, 2001. 